\title{
Análisis de la Movilidad Vehicular en el Departamento de La Guajira usando Simulación. El Caso de Riohacha y Maicao
}

\author{
Marlin A. Aarón (1), Cesar A. Gómez (1), Jeancarlos Fontalvo(1), y Armando J. Gómez (1) \\ (1) Facultad de Ingeniería, Ingeniería de Sistemas, Grupo de Investigación y Desarrollo Motivar, Universidad \\ de La Guajira, Riohacha-Colombia (e-mail: maaron@uniguajira.edu.co; caugustogomez@uniguajira.edu.co; \\ jmeyerfontalvo@uniguajira.edu.co; armandojgomez@uniguajira.edu.co)
}

Recibido Feb. 28, 2018; Aceptado May. 9, 2018; Versión final Jul. 17, 2018, Publicado Feb. 2019

\begin{abstract}
Resumen
Este trabajo analiza la movilidad vehicular en tres puntos específicos de los municipios de Riohacha y Maicao, La Guajira, en Colombia. Se utilizaron variables de flujo vehicular, como tiempos de llegada a la cola, densidad, permanencia, infracciones y otras implícitas. Se realizaron procesos de observación y descripción sistemáticos durante cuatro meses continuos en horas críticas; se identificaron y describieron las variables de flujo que impactaron los puntos seleccionados, causas y efectos, que se representan usando diagramas de influencia. Se simuló todo el proceso, usando software de alto desempeño como PTV Vissim 8 y Vensim PLE. Se reconoce que la movilidad vehicular en los puntos estudiados, genera congestión por la alta afluencia de vehículos que transitan y que las normas de tránsito vigentes allí poco ayudan a mejorar el flujo. Además de infracciones, accidentes y situaciones de intolerancia, se genera toxicidad en el ambiente por la emisión de $\mathrm{CO}_{2}$ por los vehículos en espera en cada punto crítico, asunto al que las autoridades aún no le prestan atención.
\end{abstract}

\section{Vehicular Mobility Analysis using Simulation in The Department of La Guajira: The case of Riohacha and Maicao}

\begin{abstract}
This paper analyzes the vehicular mobility in 3 specific points of the municipalities of Riohacha and Maicao, La Guajira Colombia. Variables of vehicular flow such as queue arrival times, density, permanence, infractions and other implicit ones were used. Observation and description processes are carried out over four continuous months in critical hours; the flow variables that affected the selected points, causes and effects were identified and described, which are represented using influence diagrams. The whole process was simulated, using high performance software such as PTV Vissim 8 and Vensim PLE. It is recognized that the vehicular mobility in the studied points, the congested genres due to the high frequency of vehicles that transit and that the current traffic regulations are something that don't help enough to improve the flow. In addition to infractions, accidents and situations of intolerance, toxicity is generated in the environment by the emission of $\mathrm{CO}_{2}$ by vehicles waiting at each critical point, matters to which authorities still do not pay attention.
\end{abstract}

Keywords: citizen culture; critical hours; simulation; traffic congestion; traffic regulations 


\section{INTRODUCCIÓN}

El avance en el sistema de transporte mundial ha sido determinante para la sociedad, para conectar lugares y romper la brecha de la movilización. La población actual por naturaleza hace uso de medios de transporte, para ir al trabajo, para entretenerse o movilizarse por cuestiones socioeconómicas. Todo sistema de flujo vehicular enmarca a los usuarios como factor fundamental y además el tiempo. El hecho que gran parte de la población requiera el uso de un vehículo para movilizarse a un destino fijo genera congestión vehicular, la que se agrava debido a que la población está en constante aumento (Rivadeneira, 2000). Es un problema mundial que se puede apreciar en las grandes capitales del mundo (Domper, 2003; OMM, 2008).

Este fenómeno ocurre en un lugar, un tiempo $t$ y origina efectos negativos que recaen principalmente en los usuarios, puesto que al entrar en una zona congestionada, el vehículo demora más tiempo en marcha generando así costos privados (gasolina, energía, tiempo, desgastes de los vehículos etc.) y de igual forma se generan una serie de emociones que alteran al usuario (estrés, ira, sueño). Además, si se congestiona un punto, al ocurrir un accidente o emergencia, las vías podrían estar obstruidas para las organizaciones sociales o asistenciales atentas al llamado (bomberos, ambulancias, policía, etc.), lo que provoca que se limite o se impida el objetivo de llegar al destino donde es necesitado el servicio social o ambulatorio.

Se generan problemas como pérdida de tiempo (Rivera, 2000) y costo por inactividad que puede ser aprovechada en otras labores y puede parecer extraño, pero afecta implícitamente al desarrollo económico del área (Bull y Thomson, 2002). Retardos, que se traducen en llegadas tardías al trabajo, reuniones, provocando pérdidas en negocios, acciones disciplinarias y otras medidas personales, además de lo ya mencionado sobre las dificultades en la movilidad. Cuando un carro está detenido en medio de una congestión vehicular, genera una pérdida de combustible por combustión en el aire, que se propaga gracias a las emisiones de dióxido de carbono de los vehículos y esto finalmente se traduce en daños al medio ambiente y propicia el cambio climático (Romero et al., 2006). Se le suma el estrés que produce esta congestión, lo que genera riesgos que pueden producir colisiones o accidentes. (Morales et al., 2010).

Las investigaciones y estudios realizados han permitido observar que el $47,6 \%$ de los sistemas de transporte masivo latinoamericanos se han convertido en sistemas obsoletos, a consecuencia de la baja transferencia en el recambio tecnológico, limitantes de infraestructura de las ciudades, creciente cantidad de usuarios, parque automotor deficiente y limitado (Carvajal et al., 2017). En Colombia, algunos usuarios hacen uso del servicio que el estado provee en grandes ciudades, Barranquilla, Bogotá, Cartagena, Cali, entre otras para movilizarse y el tiempo juega un factor importante. Frente a los esfuerzos por tratar de solucionar o mitigar la problemática, el tráfico vehicular es regular, (Robles et al., 2009) y en ciertas ciudades, en su mayoría en las grandes capitales ya citadas, se han propiciado soluciones; servicios de transporte masivos y buses alimentadores, así como jornadas en las que se limita el transporte de vehículos, entre otras.

Bogotá, la capital de Colombia, no es ajena a los problemas de congestión vehicular, (Pacheco y Franco, 2009) y es una de las ciudades donde se siente con fuerza, al punto que la movilidad de los vehículos se detiene o se ralentiza por tiempos prolongados por razón de los embotellamientos, identificados estos como puntos en donde se genera congestión vehicular y se detiene la movilidad por intervalos de tiempo.. Un artículo del periódico El Espectador (Colombia) (Navarrete, 2014) en el que se habla sobre las causas, enfatiza la cultura ciudadana, el crecimiento de automóviles que es más rápido que el desarrollo de rutas y la carencia de vías alternativas que promuevan un mejor flujo. La conducta y disposición de los conductores, como la respuesta popular al sistema, el poco respeto por las leyes y normas de tránsito, no ayudan al buen funcionamiento del sistema vehicular.

Riohacha y Maicao, son ciudades del Departamento de La Guajira en Colombia, que tienen un crecimiento paulatinamente lento en infraestructura de vías, en oposición al crecimiento exponencial de la población (Capel, 2009) y el uso de medios de transporte terrestres propietarios y de servicio público. El distrito de Riohacha y el municipio de Maicao se encuentran en una crítica situación en temas de movilidad vehicular, con un alto flujo de vehículos en las calles durante todo el día lo que Ortúzar (2002) denomina congestión vehicular. Esta propicia intolerancia por parte de los conductores y peatones, imprudencias e incumplimiento de las normas de tránsito, incremento en los tiempos de viajes, incertidumbre de horarios de llegada, aumento del consumo de combustible y de costos de operación, polución, en comparación con el flujo vehicular libre.

La comunidad, las empresas aseguradoras de accidentes de tránsito y los gremios de transportadores legalmente constituidos, han denunciado a través de los medios de comunicación local, los accidentes que ocurren en razón de las dificultades que se presentan en varios puntos de estos municipios para movilizarse, ya sea en vehículo o a pie. En las llamadas horas pico, definidas entre las 7:00 am - 8:15 am, 12:00 m -1:00 pm y de 5:45 pm - 7:00 pm, se observa que es difícil movilizarse de un lugar a otro. 
La ciudad de Riohacha poseía capacidades necesarias para un buen flujo de vehículos. Hoy, debido al aumento en el número de vehículos circulando, se ha llegado al embotellamiento que antes no se veía, Constantemente en varios sectores de la ciudad, se observan retrasos de los vehículos para circular en tiempos máximos de lo que lo harían en momentos de circulación con flujo normal generando congestión vehicular (Marfia y Roccetti, 2011). Se observan esfuerzos a cargo de las autoridades que se han concentrado en disminuir el flujo excesivo de vehículos, aplicando algunas medidas en puntos críticos, i) control a los vehículos mal estacionados para no obstruir el flujo vehicular, ii) semaforización para mejorar la circulación iii) días sin moto, iv) control y apoyo de la Policía Nacional y presencia de controladores de tráfico en horas pico.

En Maicao, el esfuerzo y las acciones han sido menores en el tiempo. Algunas de las autoridades municipales han sido más proclives a desarrollar acciones que otros y lo que se observa es que se han mejorado las condiciones de algunas vías y los sentidos de estas para tratar de favorecer y disminuir la congestión. Pero, la situación también es altamente entrópica. Los conductores abusan de las vías, transitan y se parquean en andenes y espacios que son para uso de los peatones, lo que hace que estos tengan que usar la calle para transitar y atravesarlas de manera atropellada lo que pone en peligro sus vidas y generan desorden. La presencia de vehículos que recogen pasajeros para ir a diferentes destinos, aumenta la peligrosidad, los accidentes, personas y vehículos. Transitar por las calles es un peligro y la calle es un caos.

Para este estudio se tomaron tres puntos cruciales, dos en la ciudad de Riohacha y uno en Maicao respectivamente. La rotonda o round point, ubicada en la Calle 15 con Carrera 6a, la intersección de la calle 15 con carrera 5 y la calle 13 con carrera 13 en Riohacha y la calle 13 con carrera 13 en Maicao. En estos lugares se ocasiona embotellamiento de tráfico. Se usa la simulación para modelar el flujo vehicular, generar conocimiento sobre las razones que explican la situación de los sectores en cuestión y aportar información soportada desde el conocimiento científico que sirva a la toma de decisiones que generen impacto positivo. Se presenta el comportamiento de los vehículos, cantidad de vehículos que hacen cola en la calle para pasar, los tiempos que demoran los vehículos en entrar y salir de la congestión, así como la representación gráfica de esta movilidad, usando diagramas de influencia, que permite presentar las relaciones entre los componentes de un sistema y el impacto que generan sus dinámicas (Aaron et al, 2016) como parte de la simulación.

El punto uno de Riohacha, calle 15 con carrera 6ª , es bastante crítico. Allí converge una vía principal de la ciudad y una vía de alto flujo de usuarios, por ser su tramo parte de carretera nacional e internacional. En ese punto se pueden apreciar los efectos del aumento vehicular. Es una construcción vial diseñada para facilitar los cruces de caminos y reducir el peligro de accidentes; se ha vuelto propenso a accidentes tanto vehicular como de peatones, lo contrario para lo que fue diseñada. Sumado a esto, la dimensión y curvatura del anillo del punto, son muy pequeños para la cantidad de vehículos que circulan por ahí, ya que la velocidad de un vehículo es inversamente proporcional a la curvatura donde se desplace, (Carrillo et al., 2015), ocasionando un pobre flujo vehicular en la zona. El otro punto, el dos, en Riohacha, está a escasos 100 metros de la rotonda en la intersección de la calle 15 con carrera 5, se genera caos vehicular. Se ven mutuamente afectados estos puntos por su relativa cercanía. Es una zona aquejada por aspectos como el cruce de dos vías principales una nacional (Calle 15) y una local (Carrera 5) damnificadas por la cercanía con la salida a zona de universidades y colegios, cercano al centro de la ciudad (zona comercial) y la presencia de locales como talleres para reparación de vehículos y cooperativas de transporte intermunicipales que ocupan dos de los cuatro carriles posibles y un sin número de transeúntes imprudentes y sin un puente peatonal que evite accidentes. La calle 15 es un importante corredor vial de la red nacional de vías de Colombia; es la carretera más importante de la región caribe nacional que atraviesa el país por la cual circulan transportes comerciales y de pasajeros. Coincide a su vez con la carrera 5 que es una vía rápida que conecta a la troncal con el centro de la ciudad. Un punto de cruce a la zona de colegios y universidades de la ciudad.

El punto 3 de análisis, la calle 13 con carrera 13 en Maicao; una zona comercial bastante transitada, los dispositivos de señalización de tránsito (semáforos) no funcionan y allí está ubicado el gremio de una cooperativa de vehículos que realizan viajes intermunicipales entre Riohacha y Maicao, cada uno de 4 puestos. Estos vehículos permanecen parqueados en una calle que no cuenta con bahías para ello, lo cual reduce el poco espacio para transitar, con un alto y permanente flujo de personas y carros.

Soportado en la realidad, experiencia y en la Teoría General de Sistemas, todo sistema llega a un punto entrópico (Arnold y Osorio, 1998; Itriago, 2011), lo que genera caos, y para este caso, el alto flujo vehicular, las infracciones, imprudencias, entre otros, puede generar que el sistema colapse totalmente. Se considera que la simulación de la realidad que se observa, en este caso colas de vehículos, es una excelente herramienta para que la ciudad pueda identificar causas y soluciones, de manera tan gráfica que sea posible comprenderla rápidamente como lo justificaron en su artículo "Simulación de sistemas de transporte público masivo" (Ortiz y Serrano, 2006) en la que expresan que un modelo de tránsito puede ser aún más interesante si es considerado como una red de colas abierta, en donde se liberan las suposiciones de modelos de redes 
de colas típicos como el de Jackson y se hace posible el empleo de distribuciones de probabilidad más adecuadas, tanto teóricas como empíricas. Lo anterior, a través de la simulación. El estudio de flujo y movilidad vehicular usando simulación posibilita una sensación de estar en el lugar de los hechos y observar el problema en una dimensión casi real. La simulación de los sistemas de transporte es el enfoque matemático y sistemático del modelo de los sistemas de transporte, (Escobar et al., 2015), como vías férreas, rutas, rotondas, mallas, etc. apoyándose en conjunto con los computadores y el software apropiado para ello.

Aunque Maicao y Riohacha no sean grandes urbes, aún en ciudades pequeñas analizar el comportamiento de los vehículos de manera manual es complejo y se realiza con gran dificultad. La simulación ayuda a definir la demanda de tráfico (Krajzewicz et al., 2012) y como los servidores, pueden ser útiles. Se pretende encontrar la raíz del problema y los efectos que producen y proveer conocimiento para la toma de decisiones. El interrogante clave es, ¿la simulación de la movilidad vehicular en cada uno de los puntos críticos podría ayudar a comprender el problema, determinar sus causas y ayudar proponer soluciones?, La simulación puede ser una herramienta poderosa para la gestión del transporte en la sociedad, permite estudiar modelos muy complicados para analizar por ser matemáticos. El modelo de simulación tiene una serie de parámetros de entrada que permiten la evaluación de numerosos escenarios (Hidalgo, 2005).

Lo más atractivo del uso de la simulación es la posibilidad de visualizar el tráfico en tiempo real, evaluar la efectividad de las estrategias locales de control de tráfico para reducir las emisiones en puntos críticos (Papson et al., 2012) y realizar predicciones condicionados por parámetros como la densidad, tipo de automóvil, velocidad, rutas alternativas, etc. "Sokolowski y Banks (2009) manifiestan que para entender la simulación es importante entender el concepto de estado de sistema, porque este grupo de variables contienen información que describe la evolución del sistema en el tiempo".

Los softwares de simulación son herramientas con alta demanda, sobre todo en planes de desarrollo urbano, donde se piensa en el máximo beneficio en calidad y planificación. La plataforma de simulación denominada PTV Vissim 8 es usada para modelos microscópicos de tráfico vehicular. Robles et al., (2009) presentan estos modelos, como la escala más pequeña para el acercamiento al análisis de los sistemas de tráfico urbano. Indican que sus variables se relacionan con el comportamiento de vehículos individuales respecto a la infraestructura y a los demás vehículos en ella. PTV Vissim 8 es una herramienta computacional que permite realizar observaciones sobre el escenario de acción de la intervención y recrear la dinámica del mismo, con las aplicaciones para el análisis del tráfico y transporte como VISSIM y SimTraffic, que ofrecen herramientas para la visualización de mapas con información 3D georreferenciada y la simulación del comportamiento de vehículos y peatones y con esto apoyar la toma de decisiones con base en escenarios plausibles (Romero et al., 2009).

El análisis de los parámetros clave con este software, permite proponer soluciones. Posterior a la introducción de datos, PTV Vissim 8 simula, crea diferentes soluciones atendiendo la problemática, nuevamente se simula para retroalimentar y finalmente Vissim devuelve datos indicando si es o no viable la solución; de ahí su importancia, todo esto sin ningún accidente. Vensim PLE es una versión educativa de Vensim, software que cuenta con herramientas poderosas para el desarrollo de modelos de simulación de gran detalle y complejidad basados en los conceptos de la dinámica de sistemas, del que usaremos los diagramas causales o de influencia.

\section{MATERIALES Y MÉTODOS}

Se realiza una investigación no experimental y de campo, en la que se llevaron a cabo procesos de observación y descripción sistemáticos durante cuatro meses continuos, 3 veces a la semana a 3 puntos críticos durante una hora de alta circulación. Dos (2) en Riohacha y uno (1) en Maicao para hacer registros históricos de flujo y densidad vehicular. Se organizaron las fases (3) reconociéndose que los servidores para atender el servicio de atravesar al otro lado de la calle, es la norma de tránsito. Se evaluó la posibilidad de seleccionar como factor metodológico los modelos de regresión lineal para estimar los tiempos de viaje de los vehículos, pero ya han comprobado anteriormente (Sabogal et al., 2015) que los modelos de regresión lineal no son buenos para predecir los tiempos de llegada porque hay violaciones a los supuestos de normalidad, independencia y homocedasticidad. Se recurre al análisis de escogencia de puntos críticos que interpretó en su artículo (Montoya, 2008) que permite atender variables e identificar similitudes para escoger puntos críticos de manera correcta.

En la fase inicial, se identificaron y describieron las variables de flujo que impactan los puntos seleccionados y se tomaron evidencias de ellas, que se registraron en una base de datos diseñada para almacenar de manera indexada por punto crítico, los datos. La selección de la hora en los puntos críticos de cada ciudad se hizo teniendo en cuenta el análisis del sitio por alto flujo vehicular, colas, vías comprometidas y el tipo de vehículos que circulaba por ellos. La recolección de datos se hizo usando un instrumento que contenía las 
siguientes variables, tiempo de llegada de los vehículos a la cola, cantidad y tipo de vehículos en la cola, tiempo en el que salían de la cola de espera e infracciones cometidas. Las variables fueron analizadas, no controladas. La hora de observación fue de 5:00 a 6.00 pm. Los días de análisis fueron lunes, miércoles y viernes.

Los observadores, 12 en total, 4 en cada punto, tomaban responsabilidad de registrar cada uno, una parte de los datos de inicio a fin durante la hora de permanencia, usando el formato, que luego servía para alimentar la base de datos que era la misma para todos los 12 observadores. Se grababa un video durante la hora de registro de datos de la actividad vehicular y de transeúntes. La fase siguiente corresponde al análisis de los datos; se calcularon promedios, definiéndose la distribución de probabilidad que aplicaba para las variables observadas. Todas las variables registradas, eran de entrada y parámetros claves para la simulación, que fue la técnica usada para generar conocimiento, representar la realidad, analizar con precisión y predecir comportamiento durante esa hora y otras horas con condiciones igual a las analizadas. Cómo variables de salida, está el tiempo de permanencia en la cola, el total de infracciones cometidas y el total de vehículos por tipo y por destino..

En la fase siguiente, después de la convergencia descriptiva, se simula el proceso, usando softwares de alto desempeño como PTV Vissim 8 y Vensim PLE, permitiendo corroborar los datos recogidos con los generados por ordenador y predecir comportamiento de las variables. Se realizaron modelos descriptivos soportados en números y representaciones gráficas usando diagramas causales o de influencia que presentan las razones expresas de la congestión vehicular en cada punto, las predicciones y propuestas de solución para reducir índice de infracciones y accidentabilidad en el sector cuestionado.

\section{RESULTADOS Y DISCUSIÓN}

El instrumento de observación se convirtió en una matriz de observación de datos, que fue mejorando su estructura en el desarrollo de la fase inicial y que sirvió para alimentar la base de datos. Los vehículos se clasificaron en livianos, cuyo peso y tamaño son adecuados para máximo 2 personas, motocicletas, mototaxis, incluso bicicletas. Pequeños, cuya altura no supera a los 2,5 metros, como camionetas, taxis, autos particulares de transporte y los vehículos grandes, cuyo proporción y peso es de más de 10 tons, camiones de carga, tractomulas, buses, etc. En la tabla 1, con datos registrados para una de las horas de observación, se presentan datos sobre vehículos, tiempo y número de infracciones cometidas.

Tabla 1: Matriz de observación de datos

\begin{tabular}{|c|c|c|c|c|c|c|c|c|c|c|}
\hline \multirow{2}{*}{$\begin{array}{c}\text { Intervalo } \\
\text { (min.) }\end{array}$} & \multicolumn{2}{|c|}{ Cantidad de vehículos en espera } & \multicolumn{2}{|c|}{ Tiempo (min.) del vehículo para } & \multicolumn{3}{|c|}{$\begin{array}{c}\text { Número de Infracciones a } \\
\text { vehículos }\end{array}$} & $\begin{array}{c}\text { Fecha } \\
\text { de Re-- } \\
\text { gistro }\end{array}$ \\
\cline { 2 - 13 } & Pequeños & Grandes & Livianos & $\begin{array}{c}\text { Llegar a la } \\
\text { cola }\end{array}$ & $\begin{array}{c}\text { Ser } \\
\text { atendido }\end{array}$ & $\begin{array}{c}\text { Salir de la } \\
\text { cola }\end{array}$ & Grandes & Medianos & Pequeños & \\
\hline $00-05$ & 0,000 & 0,000 & 0,000 & 0,000 & 0,000 & 0,000 & 0 & 1 & 0 & $4 / 6 / 2016$ \\
\hline $05-10$ & 14,000 & 0,000 & 3,000 & 2,951 & 2,000 & 4,100 & 0 & 2 & 0 & $4 / 5 / 2016$ \\
\hline $10-15$ & 4,000 & 1,000 & 2,000 & 2,545 & 8,849 & 11,653 & 0 & 0 & 1 & $4 / 5 / 2016$ \\
\hline $15-20$ & 15,000 & 0,000 & 8,000 & 4,214 & 7,662 & 12,846 & 0 & 1 & 0 & $4 / 5 / 2016$ \\
\hline $20-25$ & 10,000 & 0,000 & 4,000 & 4,871 & 9,825 & 7,289 & 0 & 0 & 0 & $4 / 5 / 2016$ \\
\hline $25-30$ & 0,000 & 0,000 & 0,000 & 4,469 & 8,426 & 5,879 & 0 & 1 & 2 & $4 / 5 / 2016$ \\
\hline $30-35$ & 10,000 & 0,000 & 2,000 & 4,053 & 7,354 & 10,441 & 0 & 1 & 1 & $4 / 5 / 2016$ \\
\hline $35-40$ & 3,000 & 1,000 & 9,000 & 3,752 & 8,077 & 4,881 & 0 & 2 & 0 & $4 / 5 / 2016$ \\
\hline $40-45$ & 7,000 & 0,000 & 0,000 & 3,807 & 8,670 & 8,179 & 0 & 0 & 2 & $4 / 5 / 2016$ \\
\hline $45-50$ & 0,000 & 0,000 & 1,000 & 6,028 & 9,061 & 10,635 & 0 & 0 & 0 & $4 / 5 / 2016$ \\
\hline $50-55$ & 10,000 & 0,000 & 0,000 & 3,250 & 9,238 & 9,243 & 0 & 1 & 3 & $4 / 5 / 2016$ \\
\hline $55-60$ & 0,000 & 0,000 & 0,000 & 2,550 & 8,423 & 10,098 & 0 & 1 & 0 & $4 / 5 / 2016$ \\
\hline TOTAL & 73 & 2 & 29 & 42,489 & 87,586 & 95,244 & 0 & 10 & 9 & $4 / 5 / 2016$ \\
\hline PROM & 6,083 & 0,167 & 2,417 & 3,541 & 7,299 & 14,653 & 0 & 0,83333 & 0,75 & $4 / 5 / 2016$ \\
\hline
\end{tabular}

En Riohacha, en la Rotonda, cada vía sirve de entrada y salida del servicio de intersección. Un flujo específico que varía en función de la hora y actividad del usuario. Para las probabilidades de la generación de la cola, se usó una distribución normal de probabilidad de Poisson, que permitía conocer cuán probable era que una cantidad $x$ de vehículos llegarán a la cola como ya lo habían demostrado con éxito (Riaño y Acero, 2005). En este caso se usó media hora, para una media de vehículos por cada 5 minutos 27.0, 16.9 y 18.3 para pequeños, grandes y livianos respectivamente. En la figura 1 se presenta como fluyen los vehículos en la rotonda, usando la simulación que provee PTV Vissim 8 y las figuras 2, 3 y 4 presenta en datos, cómo se genera la congestión y la probabilidad de que los vehículos accedan al punto crítico. 


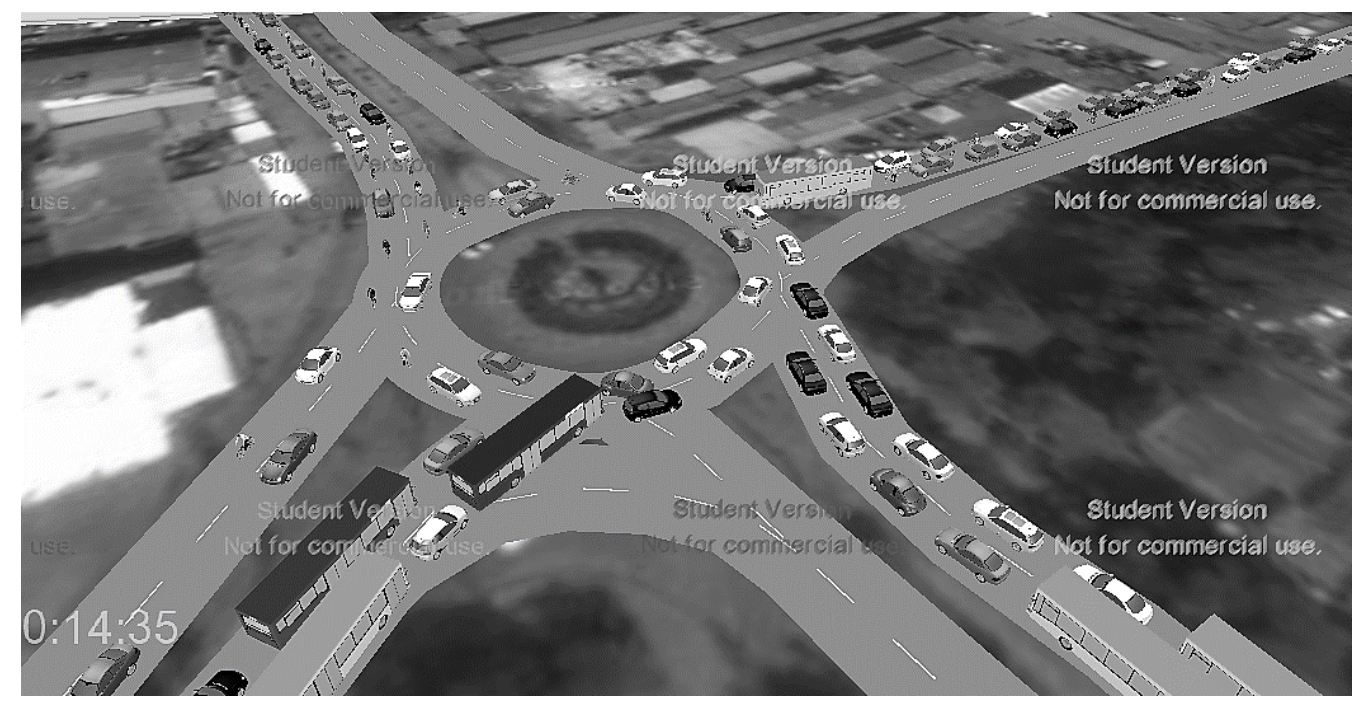

Fig. 1: Simulación por rutas y tasa de llegada (PTV Vissim 8)

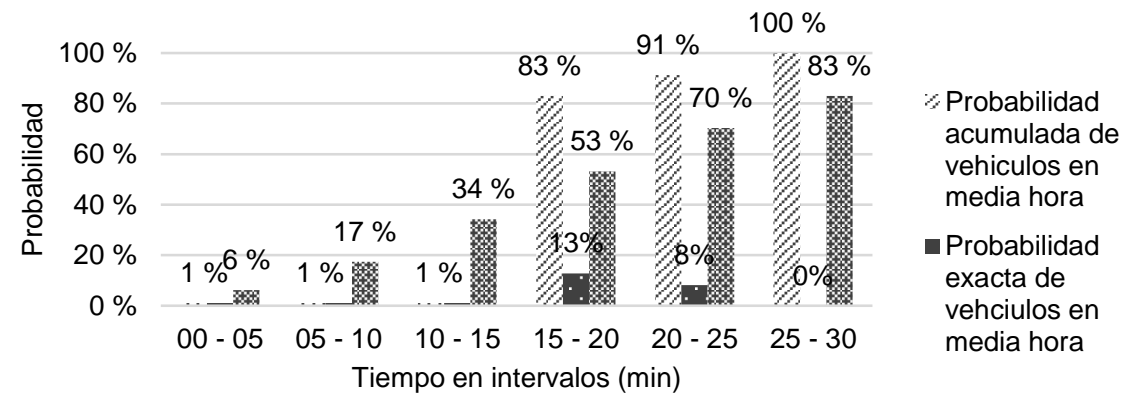

Fig. 2: Probabilidad de permanencia de vehículos pequeños en la congestión

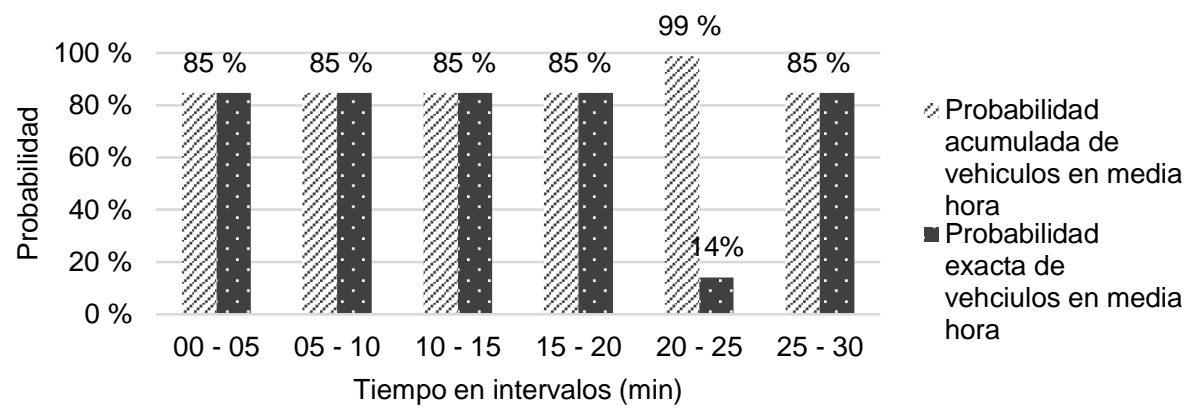

Fig. 3: Probabilidad de permanencia de vehículos grandes en la congestión

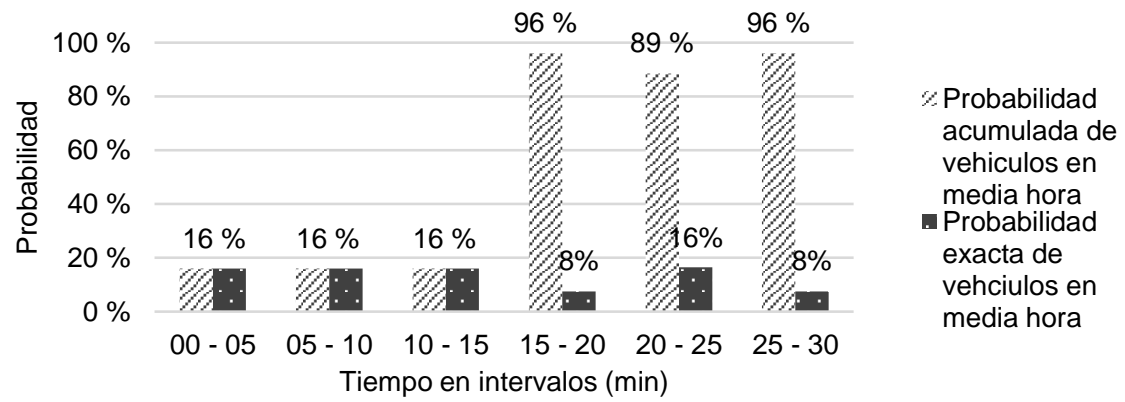

Fig. 4: Probabilidad de permanencia de vehículos livianos en la congestión. 
En este sistema, los vehículos mantienen el flujo en cada entrada de la rotonda y los vehículos pequeños y livianos aportan más del $50 \%$ en el flujo vial. La población hace uso frecuente de estos vehículos por el bajo costo de transportarse en ellos, y se observan vías repletas la mayor parte de los intervalos de tiempo estudiados. El $58 \%$ del tráfico en sentido de sur a la rotonda, está compuesto de vehículos livianos. En el caso de la vía que viene del oeste a la rotonda, el $34 \%$ del tráfico está conformado por motocicletas que circulan de forma muy rápida, $56 \%$ para vehículos pequeños y un $10 \%$ para vehículos grandes. En la vía del sentido este a la rotonda, el $32 \%$ es de vehículos livianos, los grandes ocupan el $68 \%$ restante. Esto corresponde a la vía que es carretera nacional, que tiene un alto tráfico de transporte departamental y tractocamiones. Por último, la vía de norte a la rotonda, presenta en promedio $58 \%$ de vehículos pequeños y el $30 \%$ vehículos livianos, y un reducido porcentaje para los vehículos grandes, es decir el $12 \%$, tráfico que corresponde a transporte interurbano. En cuanto a las salidas de la rotonda, la vía hacia el oeste recibe vehículos equivalentes al $14 \%$ del total de vehículos que salen de ella; mientras que la vía hacia el norte un $28 \%$. Asimismo, la vía hacia el este y el sur reciben de la rotonda $30 \%$ y $28 \%$ respectivamente. Circulan en la rotonda regularmente peatones con comportamiento imprudente; suelen transitar de forma errada las vías para pasar de un lado a otro, causando así obstrucción entre los vehículos que circulan, los cuales se ven obligados a frenar y/o disminuir la velocidad para cederle el paso, lo que causa choques imprevistos, intolerancia, además de gritos y accidentes..

El diagrama causal o de Influencias, figura 5, pretende describir el comportamiento de ese sistema de manera dinámica. Siglas: 15S: Vía de 15 por Santa Marta; 15M: Vía de 15 por Maicao; 6AM: Vía de 6A por Mercado; $6 \mathrm{AV}$ : Vía de 6A por Valledupar.

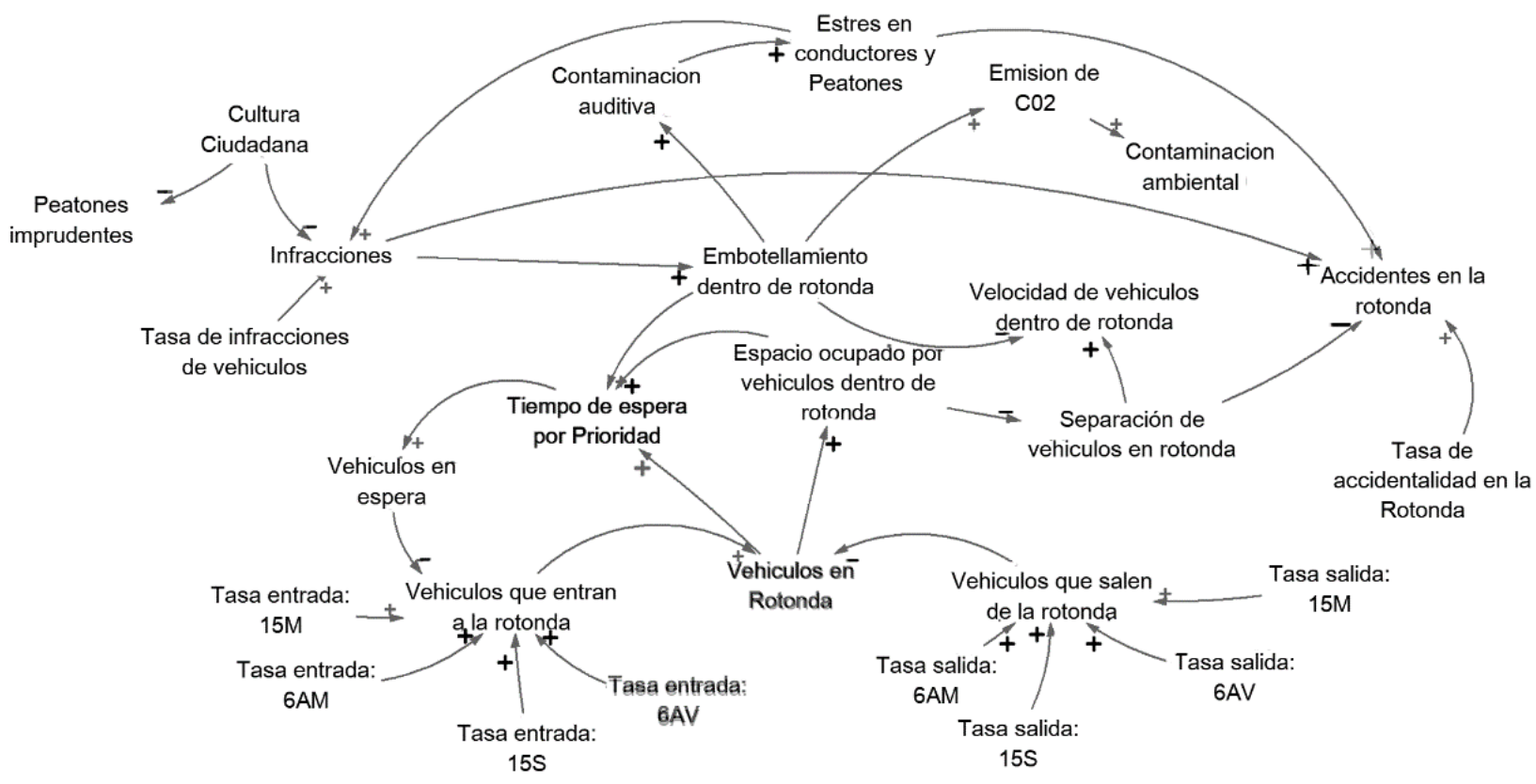

Fig. 5: Diagrama de Influencia en punto uno, realizado en Vensim PLE

El diagrama muestra que la causa del embotellamiento dentro de la rotonda está dada por los vehículos que entran por todas las entradas, norte, este, oeste, sur y quedan en espera y por las infracciones, que a su vez, son originadas por la cultura ciudadana, dado que la población por desconocimiento o de manera intencionada no respeta las normas de tránsito. La rotonda ayuda ostensiblemente a que el flujo vehicular circule, sin embargo, por intervalos de tiempo se da la congestión. En cuanto a la longitud de la cola, ésta varía en función del flujo interno de cada vía, sumado también a la cola interna de la rotonda.

En la Intersección de la calle 15 y carrera 5 (punto dos) en promedio ocurren 24 infracciones de tránsito y 8 de ellas de alto riesgo. En la figura 6 se presenta gráficamente la congestión y carros parqueados obstaculizando la vía. Esta figura fue tomada de la simulación en el software PTV Vissim 8. Se usaron 4 servidores para generar el escenario de congestión. El servidor es la opción de ingreso que permite el software para automatizar las entradas de datos que genera la vía. Son representados por las rutas de tránsito que interactúan entre sí y que para este caso se toma como regla la norma de tránsito dada por la preferencia vial a los vehículos que transitan en la calle 15.

El servidor 1 tiene como destino el sentido hacia la ciudad fronteriza de Maicao y/o la zona de colegio y universidades locales, Servidor 2 tiene muchas conexiones y gran afluencia de la ciudad; conecta a Riohacha 
con el resto del país, tiene como salida la carretera hacia la ciudad de Santa Marta. El servidor 3 viene desde el centro de la ciudad (zona comercial) y decidirá su rumbo al llegar a la intersección; el servidor 4 es el retorno hacia la zona comercial. En este orden se registra la información adquirida para simular y obtener respuestas a interrogantes sobre cómo y quiénes son los que generan la congestión vehicular en la zona. La Tabla 2 refleja la afluencia promedio de vehículos que en intervalos de una hora con relación a sus rutas y las decisiones que los conductores tomaron. Diariamente se evaluaron 3,5 horas pico en la calle 15 con carrera 5 en Riohacha; los datos promedios para la tabla 2 están dados en intervalos de 1 hora.

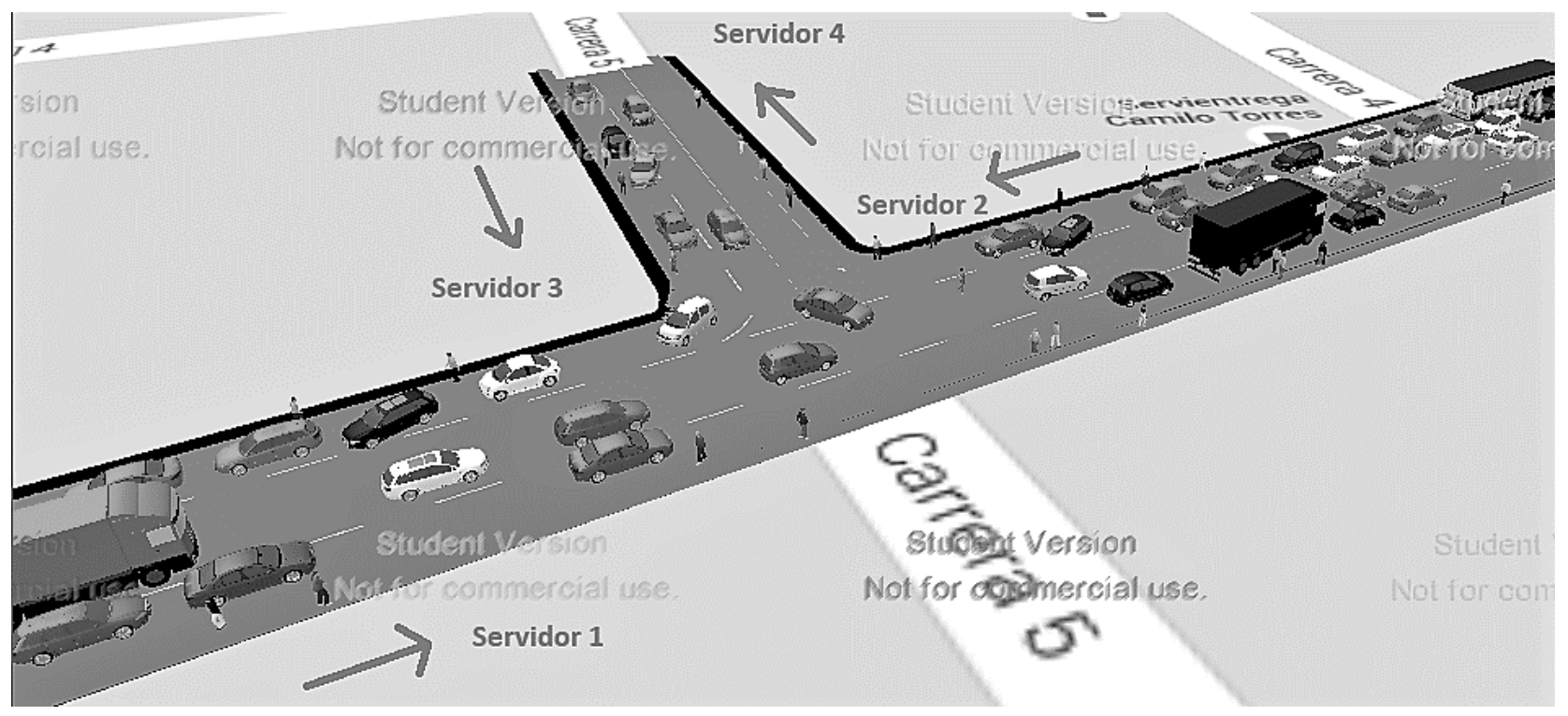

Fig. 6: Simulación en punto dos, realizada en PTV Vissim 8

Tabla 2: Flujo diario promedio de autos que transitan por hora

\begin{tabular}{|c|c|c|c|c|c|c|c|}
\hline \multicolumn{2}{|c|}{ Ingreso al Sistema } & \multicolumn{3}{c|}{ Mantienen } & \multicolumn{3}{c|}{ Desvío } \\
\hline Servidor & Nro. Autos & Vehículos & Salida & $\%$ & Vehículos & Salida & $\%$ \\
\hline 1 & 1043 & 826 & 1 & 79,19 & 217 & 4 & 20,81 \\
\hline 2 & 2051 & 1666 & 2 & 81,23 & 385 & 4 & 18,77 \\
\hline 3 & 459 & 360 & 2 & 78,43 & 99 & 1 & 21,57 \\
\hline
\end{tabular}

Se destaca que los autos que ingresan al sistema por la troncal del Caribe (servidores 1 y 2) un $80 \%$, mantienen su destino sin realizar desvíos que causan embotellamiento. El problema detectado está en vehículos que vienen del servidor 3 y por buscar comodidad a la hora de elegir rutas, toman la carrera 5ta para salir por el servidor 1 . Estos vehículos crean un caos total en la movilidad debido a que no tienen privilegio de vía y deben parar por obligación y permanecen detenidos en algunas oportunidades más de 2 minutos para cruzar; de este modo condenan a los demás vehículos permanecer en espera, generando congestión; sin ese obstáculo, transitarían con comodidad. Muchos de estos vehículos cometen infracciones para disminuir el tiempo de espera y rebasar la señal de pare. En la tabla No. 3 se presenta la duración promedio de vehículos en cola y en el sistema. Se destacan dos rutas de conflicto por su excesiva duración, de 1 a 4 y de 3 a 1.

Tabla 3: Tiempo promedio de vehículos en cola y en sistema por servidores

\begin{tabular}{|c|c|c|c|}
\hline De & $A$ & $\begin{array}{c}\text { Cola } \\
\text { (sdos.) }\end{array}$ & $\begin{array}{c}\text { Entrada/Salida } \\
\text { (sdos.) }\end{array}$ \\
\hline 1 & 1 & 8,75 & 8,58 \\
\hline 1 & 4 & 14,93 & 19,72 \\
\hline 2 & 2 & 8,88 & 13,71 \\
\hline 2 & 4 & 11,88 & 16,60 \\
\hline 3 & 1 & 23,63 & 33,67 \\
\hline 3 & 2 & 19,34 & 24,27 \\
\hline
\end{tabular}


Se propone como solución prohibir estos cruces indebidos de carril 3 a 1 presentando como alternativa la vía circunvalar aledaña que es rápida y de doble calzada. Así mismo inhabilitar el cruce del servidor 1 al 4 que interfiere de manera decisiva y se propone la ruta alterna de la carrera 6ta o la rotonda que está cercana, en buen uso. La problemática se puede entender mejor con la figura 7 que muestra a través de un diagrama de influencia, el impacto que tiene el embotellamiento y el flujo vehicular generando un caos que en ocasiones termina con la contribución de tasas de mortalidad por accidente en Colombia.

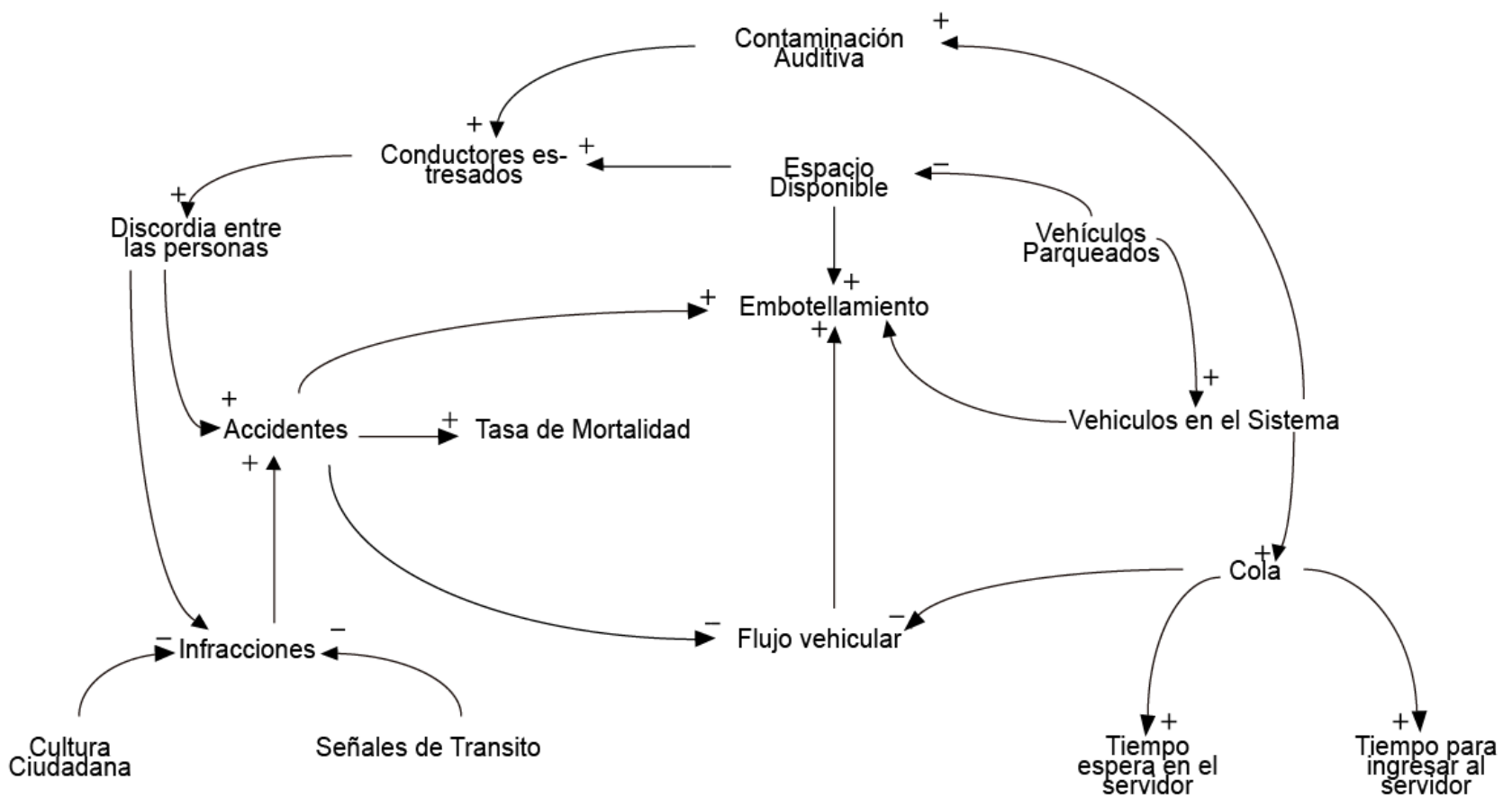

Fig. 7: Diagrama causal o de influencias del punto 2, Vensim PLE

Este diagrama causal o de influencias presenta las causas del embotellamiento o congestión vehicular; está dado por el alto flujo vehicular que genera cola, la presencia de vehículos parqueados en la zona y por las infracciones que generan accidentes, lo que evidencia una débil cultura ciudadana, que además produce accidentes, que a su vez generan más embotellamiento.

En la ciudad de Maicao, en la calle 16 con carrera 10 (punto 3), transita un alto flujo de vehículos livianos, motos y moto-car, que son motos habilitadas para transportar dos y tres pasajeros. Los resultados después de promediar la matriz de observación de datos durante 10 jornadas, se presentan en la tabla No. 4. Se consideró valioso para este caso, presentar de manera separada los resultados de vehículos livianos motos y moto-car, por cuanto en la ciudad éste el tráfico más alto de vehículos. Un alto porcentaje de estos vehículos se quedan parqueados con motor encendido esperando pasajeros.

Tabla 4: Tiempo promedio que permanecen los vehículos en la congestión

\begin{tabular}{|l|c|c|c|c|c|}
\hline & $\begin{array}{l}\text { Q promedio } \\
\text { de vehículos } \\
\text { livianos } \\
\text { motos }\end{array}$ & $\begin{array}{l}\text { Q promedio de } \\
\text { vehículo livianos } \\
\text { moto-car }\end{array}$ & $\begin{array}{l}\text { Q promedio } \\
\text { de carros } \\
\text { pequeños }\end{array}$ & $\begin{array}{l}\text { Q promedio } \\
\text { de carros } \\
\text { grandes }\end{array}$ & $\begin{array}{c}\text { Tiempo } \\
\text { promedio de } \\
\text { demora en el } \\
\text { sistema (1 h) }\end{array}$ \\
\hline Calle 16 & 1.448 & 967 & 1.333 & 276 & $7 \mathrm{~m}$ \\
\hline Carrera 10 & 816 & 554 & 609 & 27 & $1,5 \mathrm{~m}$ \\
\hline
\end{tabular}

Estos promedios están dados en unidades de vehículos. Los vehículos que transitan tienen dos opciones de destino, seguir en la misma dirección que traen o cruzar a su derecha. Estas son las rutas permitidas por las normas de tránsito. Se valoró la probabilidad que los vehículos sigan en la misma dirección o crucen a la derecha, y se usó la regla de Laplace con dos eventos y se debe elegir uno. La probabilidad queda así: $1 / 2=0,50$; el $50 \%$ de los vehículos seguirá su misma dirección, el otro $50 \%$ cruzara a la derecha.

En Maicao se observa una alta movilidad de vehículos de modelos antiguos y en pésimo estado que generan una alta contaminación auditiva la cual no puede pasar desapercibida porque causante de accidentes por descoordinación de conductores (Echeverri et al., 2011). Además de los parqueos con los vehículos encendidos que genera una alta emisión de $\mathrm{CO} 2$, es común observar carros que se dañan en la vía por su estado de deterioro y falta de mantenimiento, lo que aumenta la congestión vehicular. El $90 \%$ de los 
conductores de los vehículos priorizan la espera de los pasajeros frente a la invasión del espacio de la calle para la circulación de otros vehículos. El $10 \%$ restante utilizan los espacios para parqueo que existen. El diagrama de influencias que representa las causas de lo que ocurre en este punto, realizado en el software Vensim Ple, se presenta en la figura 8.

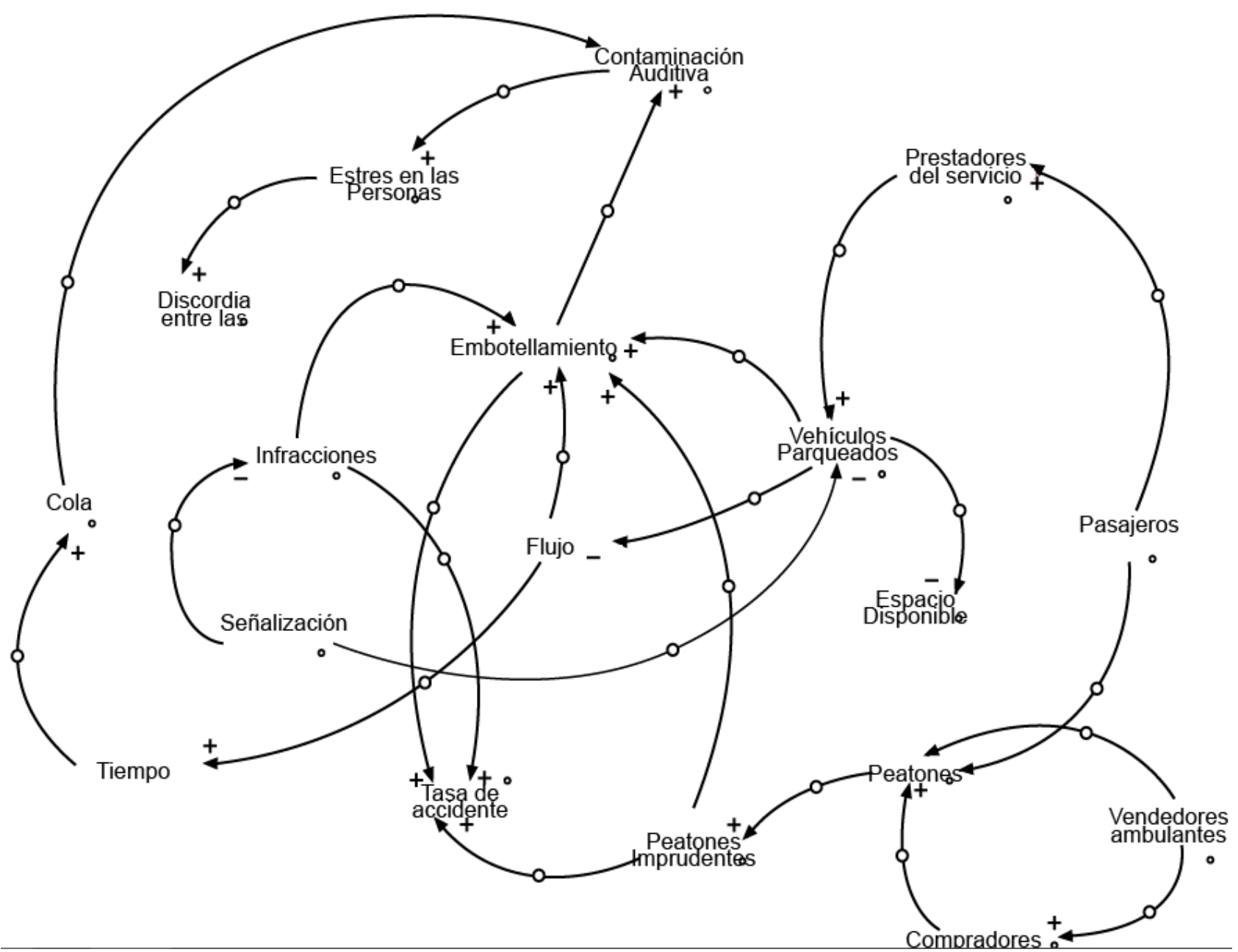

Fig. 8: Diagrama causal o de influencias, punto 3 Vensim PLE

El diagrama causal o de influencias evidencia tres aspectos que causan el embotellamiento, el flujo vehicular, alta presencia de vendedores ambulantes, vehículos mal parqueados, peatones imprudentes, infracciones, estos últimos asociados a la cultura ciudadana que no favorece una movilidad vehicular y de personas a tiempo y de manera segura. El comportamiento de los conductores y transeúntes en Maicao indican que existe una cultura ciudadana débil hacia el respeto a las normas de tránsito ya que los peatones y conductores cometen imprudencias por desconocimiento o por costumbre.

Con este estudio se intenta aportar a la disminución de los accidentes en Colombia y reducir el índice de mortalidad a través del aporte que permite la simulación de estos puntos críticos. Según el DANE el total de muertes en 2016 fue de 223.078, de las cuales 193.665 fueron de muerte natural y 29.413 personas fallecidas en accidentes de tránsito, de los cuales el $20,3 \%$ fueron hombres y el resto damas. Muertes que pudieron evitarse.

\section{CONCLUSIONES}

La alta movilidad vehicular en los puntos críticos de Riohacha y Maicao genera embotellamiento y congestión vehicular lo que demanda la inmediata intervención de las autoridades municipales para contrarrestar su impacto.

1) Tanto en Maicao como en Riohacha, la cultura ciudadana con bajo respeto a las normas de tránsito, es uno de los factores que influyen más en el desorden de la movilidad vehicular lo que genera congestión.

2) Los vehículos desbordan los promedios de espera hasta en el doble de tiempo, lo que provoca además de la congestión vehicular, un alto consumo de combustible, ruido y emisión de $\mathrm{CO}^{2}$.

3) En Riohacha y Maicao hay una movilidad de vehículos livianos como bicicletas y motos que contribuyen a 
la accidentalidad, por las imprudencias que cometen los conductores en su afán de salir pronto de la congestión.

4) En Maicao frente al embotellamiento que se genera, prima el interés individual al bien colectivo.

5) En Riohacha los vehículos ingresan a la rotonda sin importarle respetar las normas de acceso a la misma

6) En Riohacha como en Maicao, los vehículos grandes abusan de su tamaño y peso para transitar por las vías y actúan de manera imprudente.

7). El uso de la simulación y los softwares de simulación permitió el análisis en detalle de las complejas condiciones de movilidad y el tráfico.

8) Las soluciones presentadas son una alternativa que los entes territoriales deberían tener en consideración si quieren mejorar el flujo vehicular. La simulación muestra carriles en conflicto donde se presenta el mayor índice de accidentabilidad por lo que se convierte en una excelente herramienta para la toma de decisiones.

\section{REFERENCIAS}

Aarón, M., P. Choles y A. Solano, Representación del Proceso Formativo de una Institución Etnoeducativa a Través de La Técnica del Modelo Gráfico y Descriptivo Usando Diagramas de Influencia y de Forrester, doi:10.4067/S071807642016000300008, Información Tecnológica, 27(3), 81-92 (2016)

Arnold, M. y F. Osorio, Introducción a Los Conceptos Básicos de la Teoría General de Sistemas, Cinta de Moebio, 3 (1998) Bull, A. e I. Thomson, La Congestión del Tránsito Urbano: Causas y Consecuencias Económicas y Sociales, Revista de la CEPAL, 76, 109-121 (2002)

Capel, H., Las Pequeñas Ciudades en la Urbanización Generalizada y Ante la Crisis Global, ISSN: 01884611, Investigaciones Geográficas, 70, 07-32 (2009)

Carrillo J.G., J. Aramburo y R. Ortega, Modeling The Turning Speed And Car Following Behaviors of Autonomous Vehicles In a Virtual World, ISSN: 1405-7743, Ingeniería Investigación y Tecnología, 16(3), 391-405 (2015)

Carvajal L., V. Alzate e Y.F. Ceballos, Simulación de la Experiencia del Cliente en Puntos de Atención de un Sistema de Transporte Masivo Empleando Dinámica de Sistemas, ISSN: 1909-8367, Entre Ciencia e Ingeniería, 11(21), 73-81 (2017)

Domper, M., Congestión Vehicular y Tarificación Vial, ISSN: 0717-1536, Serie Informe Económico, 137, 1-35 (2003)

Echeverri C.A., D. Murillo y G. Valencia, Simulación de Ruido de Tránsito Automotor Como Herramienta para el Rediseño de Rutas de Transporte Público Colectivo en El Municipio de Medellín, ISSN: 1692-3324, Revista Ingenierías Universidad de Medellín, 10(18), 19-30 (2011)

Escobar, D., J. Giraldo y O. Tapasco, Medición de Desempeño del Sistema de Transporte Cable Aéreo de La Ciudad De Manizales En Colombia, Usando Tres Enfoques: Analítico, Simulado y De Accesibilidad Urbana, doi: 10.4067/S071807642015000600020, Información Tecnológica, 26(6), 199-210 (2015)

Hidalgo, D., Comparación de Alternativas de Transporte Público Masivo - Una Aproximación Conceptual, https://goo.gl/EdmBSK, ISSN: 0121-4993, Revista de Ingeniería, 21, 94-105 (2005)

Itriago, P.G., Caracas, Vialidad y Transporte. Una Visión Crítica Desde la Teoría del Caos, ISSN: 03039757, Politeia, 34(46), 133-173 (2011)

Krajzewicz, D., J. Erdmann, M. Behrisch y L. Bieker, Recent Development and Applications of SUMO-Simulation of Urban Mobility, https://goo.gl/AAKGmV, ISSN: 1942-261x, International Journal on Advances in Systems and Measurements, 5(3\&4), 128-138 (2012)

Marfia, G. y M. Roccetti, Vehicular Congestion Detection and Short-Term Forecasting: A New Model with Results, https://goo.gl/pk9P9e, ISSN: 0018-9545, IEEE Transactions on Vehicular Technology, 60(7), 2936-2948 (2011)

Montoya, J.R., Un Procedimiento Greedy Para El Problema de Posicionamiento de Vehículos en Sistemas Complejos de Transporte Automatizado para Manufactura, ISSN: 0123-2126, Ingeniería y Universidad, 12(1), 9-32 (2008)

Morales-Soto, N., D. Alfaro-Basso y W. Gálvez-Rivero, Aspectos Psicosociales y Accidentes en El Transporte Terrestre, https://goo.gl/Kj6MYT, ISSN: 1726-4634, Revista Peruana de Medicina Experimental y Salud Pública, 27(2), 267-272 (2010)

Navarrete, S., La Ausencia de Cultura Ciudadana Contribuye a La Congestión Vehicular, El Espectador, 9-jun. (2014)

OMM, Movilidad En Bogotá Una Verdadera Pesadilla, Observatorio de la Movilidad Metropolitana, 20-jun. (2008)

Ortiz, J.E. y L.A. Serrano, Simulación de Sistemas de Transporte Público Masivo, https://goo.gl/58FSNW, ISSN: 01205609, Ingeniería e Investigación, 26(1), 51-57 (2006)

Ortúzar, J., Displacements: Is It Possible To Reduce Vehicular Congestion? Doi: 10.4067/S0717-69962002005200005, ARQ (Santiago), (52), 7-9 (2002)

Pacheco, J., J.F. Franco y E. Behrentz, Caracterización De Los Niveles De Contaminación Auditiva En Bogotá: Estudio Piloto, https://goo.gl/S1KcNC, ISSN: 0121-4993, Revista de Ingeniería, 30, 72-80 (2009) 
Papson, A., S. Hartley y K.L. Kuo, Analysis Of Emissions At Congested And Uncongested Intersections With Motor Vehicle Emission Simulation 2010, doi: 10.3141/2270-15, Transportation Research Record: Journal of the Transportation Research Board, 22(70), 124-131 (2012)

Riaño G. y J.C. Acero, Implementación y Análisis de Un Modelo Estocástico de Despacho de Vehículos de Transporte Masivo, ISSN: 0121-4993, Revista de Ingeniería, 21, (2005)

Rivadeneira, S., América Latina y el Caribe: Crecimiento Económico Sostenido, Población y Desarrollo, 2ª Ed., 1-59, CEPAL - SERIE Población y Desarrollo, Santiago de Chile (2000)

Rivera, V.I., Llegando Tarde Al Compromiso: La Crisis del Transporte en La Ciudad De México, 1ª Ed., El Colegio De México, Ciudad de México, México (2000)

Robles, D., P. Ñañez y N. Quijano, Control y Simulación de Tráfico Urbano En Colombia: Estado Del Arte, goo.gl/f5Vbma, ISSN: 0121-4993, Revista de Ingeniería, 29, 59-69, Retrieved enero 20, 2017 (2009)

Romero, W., J.T. Hernández, J.C. Ibarra y S. Ordoñez, Plataforma de Simulación y Visualización Para el Apoyo al Análisis y Toma de Decisiones en Proyectos de Movilidad Urbana, ISSN: 0121-4993, Revista de Ingeniería, 1(29) (2009)

Romero, M., F. Olite y M. Álvarez, La Contaminación del Aire: Su Repercusión Como Problema de Salud, https://goo.gl/bzQqeV, ISSN 1561-3003, Revista Cubana de Higiene y Epidemiología, 44(2) (2006)

Sabogal, O.A., J.D. Hincapié, J.J. Santa, J.W. Escobar, Modelos de Regresión Lineal para Estimación de Tiempos de Viaje En Sistemas de Transporte Masivo, ISSN: 0124-8170, Ciencia e Ingeniería Neogranadina, 25(1), 77 - 89 (2015)

Sokolowski, J.A. y C.M. Banks, Principles of Modeling and Simulation: A Multidisciplinary Approach, 1ª Ed., Wiley, Estados Unidos (2009) 